\title{
PCSK9 Inhibitors and Neurocognitive Adverse Drug Reactions: Analysis of Individual Case Safety Reports from the Eudravigilance Database
}

\author{
Gabriella di Mauro ${ }^{1,2} \cdot$ Alessia Zinzi ${ }^{1,2} \cdot$ Cristina Scavone $^{1,2}(1) \cdot$ Annamaria Mascolo ${ }^{1,2} \cdot$ Mario Gaio $^{1,2}$. \\ Liberata Sportiello $^{1,2} \cdot$ Carmen Ferrajolo $^{1,2}$. Concetta Rafaniello ${ }^{1,2} \cdot$ Francesco Rossi $^{1,2} \cdot$ Annalisa Capuano $^{1,2}$
}

Accepted: 9 November 2020 / Published online: 22 December 2020

(c) The Author(s) 2020

\begin{abstract}
Introduction Proprotein convertase subtilisin/kexin type 9 inhibitors (PCSK9Is) were associated with a risk of neurocognitive adverse drug reactions (ADRs).

Objective We aimed to investigate the occurrence of neuropsychiatric ADRs related to PCSK9Is.

Methods We analyzed Individual Case Safety Reports (ICSRs) sent through the European pharmacovigilance database that reported alirocumab or evolocumab as the suspected drug and at least one neurological or psychiatric ADR. The reporting odds ratio (ROR) was computed to compare the probability of reporting ICSRs with neuropsychiatric ADRs between alirocumab, evolocumab and statins.

Results Overall, 2041 ICSRs with alirocumab and/or evolocumab as the suspected drug described the occurrence of neuropsychiatric ADRs. The most reported preferred terms for both drugs were headache, insomnia and depression. No difference between alirocumab and evolocumab was observed for the RORs of ICSRs with ADRs belonging to the System Organ Classes (SOCs) 'Nervous system disorders' or 'Psychiatric disorders' (ROR 1.02, 95\% confidence interval 0.91-1.14; and $1.12,95 \%$ CI $0.94-1.34$, respectively), while evolocumab and alirocumab had a higher reporting probability of ICSRs with ADRs belonging to the SOC 'Nervous system disorders' compared with atorvastatin and fluvastatin. A lower reporting probability was instead found for ICSRs with ADRs belonging to the SOC 'Psychiatric disorders' for evolocumab and alirocumab versus simvastatin, pravastatin and rosuvastatin.

Conclusion Our results demonstrated that $22.7 \%$ of all ICSRs reporting alirocumab or evolocumab as suspect drugs described the occurrence of neuropsychiatric ADRs. The ROR showed that evolocumab and alirocumab had a higher reporting probability of neurological ADRs compared with statins. Further data from real-life contexts are needed.
\end{abstract}

\section{Introduction}

Gabriella di Mauro, Alessia Zinzi and Cristina Scavone share first authorship.

Francesco Rossi and Annalisa Capuano are both lead authors.

Electronic supplementary material The online version of this article (https://doi.org/10.1007/s40264-020-01021-3) contains supplementary material, which is available to authorized users.

Cristina Scavone

cristina.scavone@unicampania.it

1 Department of Experimental Medicine, University of Campania "Luigi Vanvitelli", Naples, Italy

2 Regional Centre for Pharmacovigilance, Campania Region, Naples, Italy
For decades, statins have represented the mainstay of the pharmacological treatment of hypercholesterolaemia, having been shown to reduce low-density lipoprotein-cholesterol (LDL-C) levels and the incidence of atherosclerotic cardiovascular disease [1]. Apart from the decrease in hepatic intracellular cholesterol, statins are able to induce an increase in nuclear translocation of sterol-regulatory element binding protein-2 (SREBP-2) that activates low-density lipoprotein receptors (LDLRs) and proprotein convertase subtilisin/kexin type 9 (PCSK9) [2].

PCSK9 is an enzyme encoded by the PCSK9 gene in humans on chromosome 1 . Studies have demonstrated that mutations in the PCSK9 gene caused autosomal dominant hypercholesterolaemia, and that PCSK9 plays a crucial role in the lifecycle of the LDLR $[3,4]$. Thus, the discovery of PCSK9 in 2003 identified a new target for drug intervention, 


\section{Key Points}

Safety reports related to alirocumab and evolocumab and the occurrence of neuropsychiatric adverse drug reactions (ADRs) were retrieved from the Eudravigilance database and then analyzed.

ADRs most commonly identified for alirocumab and evolocumab were headache, insomnia and depression. The statistical analysis applied revealed no difference between alirocumab and evolocumab and the probability of neuropsychiatric ADRs.

Further data from real-life contexts needs to be collected in order to better characterize the neurocognitive safety profile of alirocumab and evolocumab.

leading to the development of PCSK9 inhibitors (PCSK9Is; alirocumab and evolocumab) as novel treatment options in modern lipidology $[3,5]$. Both drugs obtained marketing approval in European Union (EU) countries in 2015. Publication of the results of the FOURIER and ODISSEY OUTCOMES studies, two large-scale randomized clinical trials involving PCSK9Is, brought the use of these drugs in LDLC-lowering therapy a step further $[6,7]$. Indeed, in both trials, an incremental reduction in LDL-C of $>50 \%$ from baseline was observed, with no major safety concerns over the trials' median follow-up (2.2 and 2.8 years, respectively).

Currently, alirocumab is indicated in adults with primary hypercholesterolaemia (heterozygous familial and nonfamilial) and mixed dyslipidaemia, either in combination with a statin or alone in patients who are statin-intolerant. Instead, evolocumab is indicated in adults with hypercholesterolaemia (heterozygous familial and non-familial) and mixed dyslipidaemia (alone or in combination with other lipid-lowering therapies) and in adults and adolescents with homozygous familial hypercholesterolaemia (in combination with other lipid-lowering therapies). Based on data from epidemiological studies demonstrating a correlation between the reduction of plasma LDL-C levels and a reduction of cardiovascular risk [8], both drugs have obtained the indication for the reduction of cardiovascular risk in adults with established atherosclerotic cardiovascular disease.

Nowadays, some questions remain to be answered, especially those related to the safety profile of PCSK9Is, in terms of immunogenicity, diabetes, and neurocognitive adverse events. With regard to immunogenicity and diabetes, longterm studies have suggested the absence of significant risk of these events $[9,10]$. Neurocognitive adverse events associated with the lipid-lowering treatments remains an area of debate. Postmarketing surveillance reports, observational studies, and some small randomized trials on statins [11, 12] have suggested that these drugs (or the low levels of LDL-C resulting from their use) may be associated with impaired cognitive function. These data led the US FDA to issue a warning in 2012. In 2014, the FDA directed developers of PCSK9Is to monitor neurocognitive adverse effects and consider neurocognitive testing in at least a subset of participants in ongoing late-stage trials [13]. Furthermore, a recent preclinical study reported that cognitive impairment observed in patients with Alzheimer's disease could be associated with cholesterol metabolism alterations, which could involve PCSK9. Indeed, authors found that PCSK9 levels differ between control and Alzheimer's disease brains [14]. On the other hand, it should be highlighted that cardiovascular risk itself plays an important role in the etiology of neurocognitive diseases [15]. In fact, a high cardiovascular risk can cause morphological alterations of brain structures, and can consequently lead to more serious conditions, such as cerebrovascular disease, cognitive decline, and dementia [16].

Based on literature data and regulatory agency communications reporting a risk of neurocognitive adverse events associated with PCSK9Is [17-22], we aimed to describe neurological and psychiatric adverse drug reactions (ADRs) reported for evolocumab and alirocumab in the Eudravigilance database. We also aimed to assess the reporting odds ratio (ROR) and $95 \%$ confidence interval (CI), as well as the Chi-square test, of drugs under evaluation to evaluate if they have a lower/higher probability of reporting Individual Case Safety Reports (ICSRs) with neurological or psychiatric adverse events in a direct comparison or compared with statins.

\section{Methods}

\subsection{Data Source}

Data on ICSRs with a PCSK9I as the suspected drug were retrieved from the website of suspected ADRs (www.adrre ports.eu) of the European pharmacovigilance database (Eudravigilance) for the period January 2015 to March 2020. Eudravigilance is a system used for managing and analyzing information on suspected adverse reactions related to drugs, which have been authorized or are being studied in clinical trials in the European Economic Area (EEA). The Eudravigilance database is maintained by the European Medicines Agency (EMA) on behalf of the EU medicines regulatory network. Specifically, Eudravigilance contains all ICSRs related to either drugs or vaccines that are reported by a healthcare professional (HCP) or non-HCP to an EU national competent authority or a marketing authorization holder. These data are publicly available for transparency through the EMA website (www.adrreports.eu). 


\subsection{Selection of Individual Case Safety Reports with Line Listing}

All ICSRs that reported alirocumab or evolocumab as the suspected drug, and that reported at least one neurological or psychiatric ADR, were retrieved using the line-listing function. To identify ICSRs that reported neurological or psychiatric ADRs, we use the Medical Dictionary for Regulatory Activities (MedDRA ${ }^{\circledR}$ ) System Organ Classes (SOCs) 'Nervous system disorders' or 'Psychiatric disorders'.

\subsection{Data Analyses}

Information on patient characteristics (age and sex), adverse event (outcome and seriousness), therapeutic indication, primary source qualification, primary source country for regulatory purposes, number of suspected drugs other than the PCSK9I, and number of concomitant drugs were provided for all ICSRs and separately for ICSRs with evolocumab or alirocumab.

A case was defined as 'serious' when the ADR (1) results in death, (2) is life-threatening, (3) requires hospitalization or prolongation of existing hospitalization, (4) results in persistent or significant disability/incapacity, (5) is a congenital anomaly/birth defect, or (6) results in some other medically important conditions.

The outcome of ADRs was classified as 'recovered/ resolved', 'recovering/resolving', 'recovered/resolved with sequelae', 'not recovered/not resolved', 'fatal' and 'unknown', and was reported for ADRs belonging to the 'Nervous system disorders' or 'Psychiatric disorders'. If a case reported more than one ADR belonging to the same SOC or the Standardized MedDRA Query (SMQ), but with different outcomes, the outcome with the lower level of resolution was chosen for classification.

We also analyzed the annual trend in ICSRs of evolocumab and alirocumab-induced neurological and psychiatric ADRs. Neurological and psychiatric ADRs were tabled for evolocumab and alirocumab.

Finally, the ROR and 95\% CI, as well as the Chi-square test, were computed to compare the probability of reporting ICSRs with ADRs belonging to the SOCs 'Nervous system disorders' or 'Psychiatric disorders' between alirocumab and evolocumab, using evolocumab as the reference drug, and between evolocumab or alirocumab and statins (atorvastatin, simvastatin, pravastatin, rosuvastatin or fluvastatin).

\subsection{Compliance with Ethical Standards}

Safety data deriving from the spontaneous reporting system are anonymous and are in compliance with the ethical standards; therefore, no further ethical measures were required.

\section{Results}

From January 2015 to March 2020, 8987 ICSRs reporting alirocumab or evolocumab as suspect were sent to the Eudravigilance database (data not shown). Among these ICSRs, 2041 (22.7\%) described the occurrence of ADRs belonging to the SOCs 'Nervous system disorders' and 'Psychiatric disorders', of which 856 related to alirocumab and 1231 related to evolocumab (Table 1). As reported in Fig. 1, starting from 2015 and until 2019, for both drugs a constant increase in ICSR reporting was observed; it should be specified that data relating to 2020 are incomplete (updated 30 March 2020).

The sum of ICSRs related to alirocumab and evolocumab exceeds the total number of ICSRs $(n=2087)$ since 46 ICSRs reported both PCSK9s as suspect drugs. Overall, those ICSRs $(n=2041)$ covered 3170 ADRs, of which 2381 belonged to the SOC 'Nervous system disorders' and 789 belonged to the SOC 'Psychiatric disorders' (Table 2).

As reported in Table 1, the median age of patients who experienced ADRs was 66 years (interquartile range 59-73) and $55 \%$ were female. Regarding distribution by seriousness, $66.6 \%$ of ICSRs reported ADRs that were considered as serious, while $33.4 \%$ reported ADRs that were classified as not serious.

For the majority of ADRs, both neurological and psychiatric, the outcome was not reported (50\%), and was unfavourable (not recovered/not resolved or death) for $16.7 \%$ and $18.2 \%$ of ADRs belonging to the SOCs 'Psychiatric disorders' and 'Nervous system disorders', respectively. Lastly, the outcome was favourable (recovered or recovering) for $36.2 \%$ and $32.2 \%$ of ADRs belonging to the SOCs 'Psychiatric disorders' and 'Nervous system disorders', respectively (Fig. 2).

The primary source qualification was mainly represented by HCPs (72.3\%), while the primary source country for regulatory purposes was the non-EEA (55.3\%). In the majority of ICSRs (94\%), no suspected drugs other than PCSK9Is were reported. A higher percentage of ICSRs (61.1\%) did not report any concomitant medications (Table 1). Among ICSRs reporting concomitant medications (38.9\%), the most common reported medications were acetylsalicylic acid ( $n=313)$, ezetimibe $(n=181)$, levothyroxine $(n=120)$, clopidogrel $(n=113)$, atorvastatin $(n=84)$ and rosuvastatin $(n=73)$ [data not shown].

Regarding the distribution of ICSRs by therapeutic indications (Table 3), both alirocumab and evolocumab were mainly used for the treatment of 'Lipid metabolism disorders' (579/856 and 870/1232, respectively), followed by 'Cardiovascular disorders' (55/856 and 123/1232, respectively) and 'Other therapeutic indications' (7/856 and 
Table 1 Demographic and clinical characteristics of Individual Case Safety Reports reporting ADRs related to the System Organ Class "Nervous system disorder" or "Psychiatric disorders" and having ali-

\begin{tabular}{|c|c|c|c|c|}
\hline Variable & Level & All ICSRs $[n=2041]$ & $\begin{array}{l}\text { ICSRs reporting ali- } \\
\text { rocumab as the suspect drug } \\
{[n=856]}\end{array}$ & $\begin{array}{l}\text { ICSRs reporting evo- } \\
\text { locumab as the suspect drug } \\
{[n=1231]}\end{array}$ \\
\hline Age, years & Median (IQR) & $66(59-73)$ & $67(59-73)$ & $66(58-72)$ \\
\hline \multirow[t]{3}{*}{ Sex } & Female & $1121(55)$ & $475(55.5)$ & $668(54.3)$ \\
\hline & Male & $864(42.3)$ & 357 (41.7) & $525(42.6)$ \\
\hline & Missing & $56(2.7)$ & $24(2.8)$ & $38(3.1)$ \\
\hline \multirow[t]{2}{*}{ Seriousness } & Serious & $1359(66.6)$ & $556(65)$ & $835(67.8)$ \\
\hline & Not serious & $682(33.4)$ & $300(35)$ & $396(32.2)$ \\
\hline \multirow[t]{2}{*}{ Primary source qualification } & Healthcare professional & $1475(72.3)$ & $433(50.6)$ & $1078(87.6)$ \\
\hline & Non-healthcare professional & $566(27.7)$ & $423(49.4)$ & $153(12.4)$ \\
\hline \multirow{2}{*}{$\begin{array}{l}\text { Primary source country for } \\
\text { regulatory purposes }\end{array}$} & European Economic Area & $912(44.7)$ & $389(45.4)$ & $551(44.8)$ \\
\hline & $\begin{array}{l}\text { Non-European Economic } \\
\text { Area }\end{array}$ & $1129(55.3)$ & $467(54.6)$ & $680(55.2)$ \\
\hline \multirow{6}{*}{$\begin{array}{l}\text { Suspected drug(s) other than } \\
\text { PCSK9I }\end{array}$} & 0 & $1917(94)$ & $790(92.3)$ & $1128(91.6)$ \\
\hline & 1 & $83(4)$ & $53(6.2)$ & $71(5.8)$ \\
\hline & 2 & $24(1.2)$ & $11(1.3)$ & $17(1.4)$ \\
\hline & 3 & $6(0.3)$ & - & $6(0.5)$ \\
\hline & 4 & $5(0.2)$ & - & $5(0.4)$ \\
\hline & $\geq 5$ & $6(0.3)$ & $2(0.2)$ & $4(0.3)$ \\
\hline \multirow[t]{6}{*}{ Concomitant drug(s) } & 0 & $1246(61.1)$ & $527(61.6)$ & 749 (60.9) \\
\hline & 1 & $148(7.3)$ & $52(6.1)$ & $96(7.8)$ \\
\hline & 2 & $119(5.8)$ & $44(5.1)$ & $79(6.4)$ \\
\hline & 3 & $84(4.1)$ & $40(4.7)$ & $46(3.7)$ \\
\hline & 4 & 79 (3.9) & $37(4.3)$ & $44(3.6)$ \\
\hline & $\geq 5$ & $365(17.8)$ & $156(18.2)$ & $217(17.6)$ \\
\hline
\end{tabular}

Data are expressed as $n(\%)$ unless otherwise specified

ICSRs Individual Case Safety Reports, IQR interquartile range, PCSK9I proprotein convertase subtilisin/kexin type 9 inhibitor

$11 / 1232$, respectively). Lastly, in 635 ICSRs, the therapeutic indication was not reported.

As reported in Table 2, alirocumab was associated with 975 ADRs belonging to the SOC 'Nervous system disorders' and with 321 ADRs belonging to the SOC 'Psychiatric disorders'. The most commonly reported Preferred Terms (PTs) for alirocumab among the SOC 'Nervous system disorders' were headache $(14.05 \%)$, dizziness $(12.82 \%)$, cerebrovascular accident $(6.15 \%)$, paresthaesia $(4.41 \%)$, memory impairment $(3.79 \%)$, gait disturbance $(3.49 \%)$, hypoesthesia $(2.87 \%)$, and amnesia $(2.15 \%)$. On the other hand, the most common reported PTs for alirocumab among the SOC 'Psychiatric disorders' were insomnia (11.84\%), depression $(9.66 \%)$, confusional state $(8.72 \%)$, depressed $\operatorname{mood}(6.54 \%)$, anxiety $(6.23 \%)$, sleep disorders $(5.61 \%)$, and disturbance in attention (4.67\%) (Table 2). Evolocumab was instead associated with 1406 ADRs belonging to the SOC 'Nervous system disorders' and to 468 ADRs belonging to the SOC 'Psychiatric disorders'. Specifically, the most commonly reported PTs for evolocumab among the SOC rocumab or evolocumab as suspect drugs sent through the Eudravigilance database from January 2015 to March 2020 


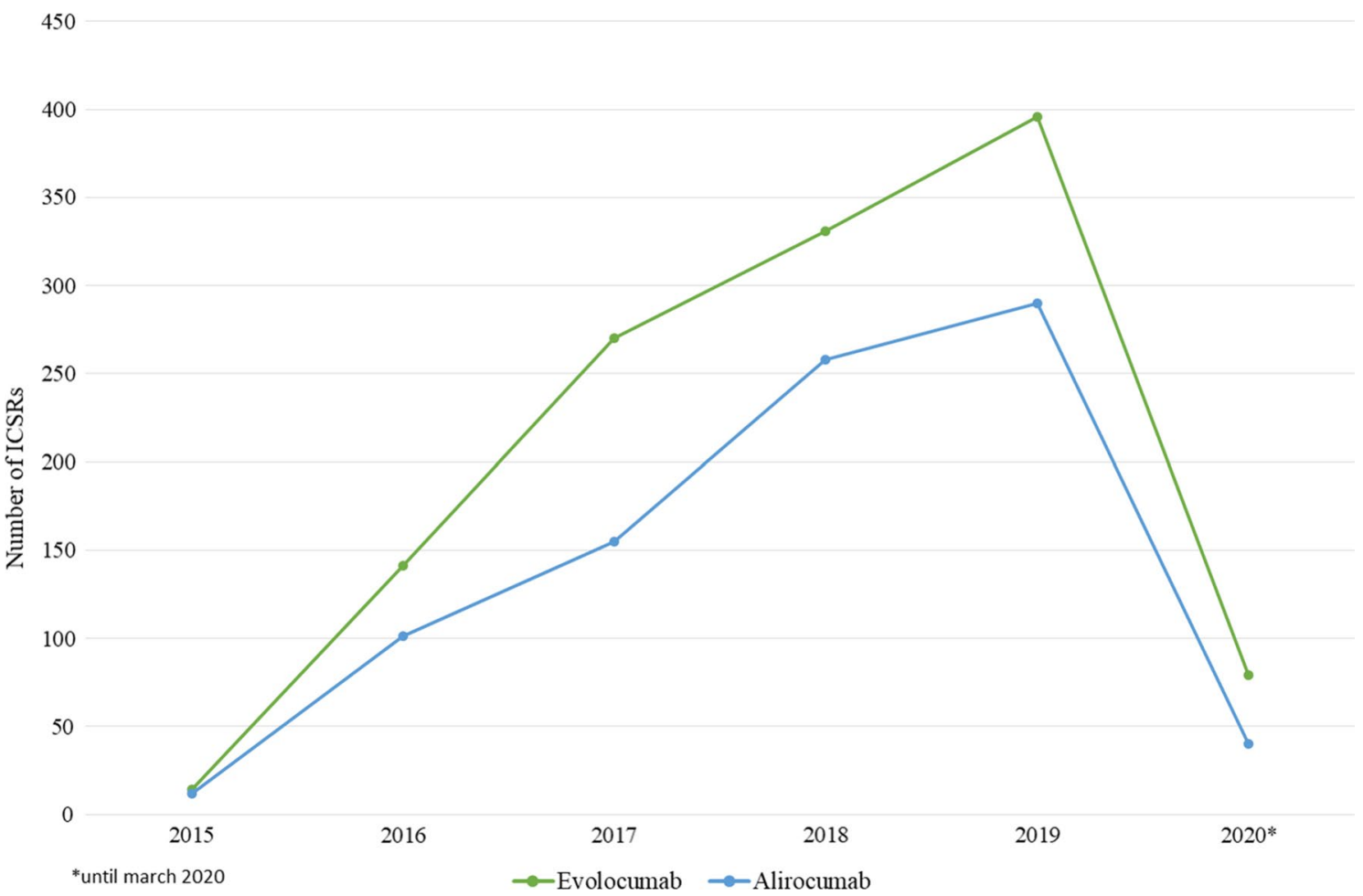

Fig. 1 Distribution of Individual Case Safety Reports having alirocumab or evolocumab as suspect drugs by year (2015-2020)

(ROR 1.55, 95\% CI 1.44-1.68; and ROR 1.58, 95\% CI $1.45-1.74$, respectively) and fluvastatin (ROR $1.49,95 \%$ CI 1.25-1.77; and ROR 1.51, 95\% CI 1.27-1.82, respectively) (Table 5). A lower reporting probability was instead found for ICSRs with ADRs belonging to the SOC 'Psychiatric disorders' for evolocumab and alirocumab versus simvastatin (ROR 0.56, 95\% CI 0.49-0.64; and ROR 0.63, 95\% CI 0.54-0.73, respectively), pravastatin (ROR 0.61, 95\% CI $0.51-0.73$; and ROR $0.69,95 \%$ CI $0.57-0.83$, respectively), and rosuvastatin (ROR 0.68, 95\% CI 0.60-0.78; and ROR $0.76,95 \%$ CI 0.66-0.89, respectively) (Table 6).

\section{Discussion}

In this study, we investigated the safety profile of PCSK9Is, in terms of neurological and psychiatric ADRs through the analysis of data from the Eudravigilance database. The choice to evaluate these specific safety concerns has been driven by scientific evidences that demonstrated a higher risk of neurocognitive adverse events with these drugs [17-22]. Indeed, according to the literature, it is biologically plausible that lipid-lowering therapies could impact brain function, causing cognitive adverse effects, and also considering that about $25 \%$ of the body's cholesterol is found in the brain [23]. Furthermore, cholesterol is the principal component of myelin, a fatty sheath that serves an essential role in cellular signalling and blood-brain barrier (BBB) integrity [24]. Despite these evidences, currently no studies have demonstrated a causal association between central nervous system cholesterol reductions and functional impairment, while factors such as coronary heart disease and age are independent risk factors for Alzheimer's disease and many other age-related conditions associated with cognitive decline [25]. Data on the role of PCSK9 in the clearance of apolipoprotein E are quite conflicting. Indeed, a study reported that PCSK9 is able to modulate the degradation of the $\beta$-site amyloid precursor protein cleaving enzyme 1 (BACE), which is involved in the generation of the amyloid $\beta$-peptides [26], leading to an increase in BACE1 levels and total amyloid $\beta$ in the brain of PCSK9 knockout mice; however, these results were not confirmed in another study [27].

In our study, we found that 2041 ICSRs reporting alirocumab or evolocumab as suspect, and ADRs referring to the SOCs 'Nervous system disorders' and 'Psychiatric disorders' were sent to the Eudravigilance database. From 2015 until 2019, for both drugs there was a constant increase in ICSR reporting that achieved a peak in 2019. The increase in ADR reporting is probably related to the increased utilization of both PCSK9Is. Indeed, these drugs obtained marketing authorization in 2015, becoming gradually available across EU and non-EU countries for use in clinical practice, and their use has constantly increased, as demonstrated by several studies [19, 28-31]. 
Table 2 Distribution of Individual Case Safety Reports having alirocumab or evolocumab as suspect drugs, by System Organ Classes and Preferred Terms

\begin{tabular}{|c|c|c|}
\hline System Organ Class & $\begin{array}{l}\text { Alirocumab } \\
{[\text { no. of ICSRs }=856]}\end{array}$ & $\begin{array}{l}\text { Evolocumab } \\
\text { [no. of ICSRs = 1231] }\end{array}$ \\
\hline Nervous system disorder [no. of ADRs $=2381]$ & [no. of $A D R s=975]$ & [no. of $A D R s=1406$ ] \\
\hline Headache & $137(14.05)$ & $249(17.71)$ \\
\hline Dizziness & $125(12.82)$ & $183(13.02)$ \\
\hline Cerebrovascular accident & $60(6.15)$ & $77(5.48)$ \\
\hline Memory impairment & $37(3.79)$ & $75(5.33)$ \\
\hline Paraesthesia & $43(4.41)$ & $66(4.69)$ \\
\hline Amnesia & $21(2.15)$ & $50(3.56)$ \\
\hline Hypoaesthesia & $28(2.87)$ & $35(2.49)$ \\
\hline Gait disturbance & $34(3.49)$ & $27(1.92)$ \\
\hline Cognitive disorder & $19(1.95)$ & $29(2.06)$ \\
\hline Burning sensation & $20(2.05)$ & $27(1.92)$ \\
\hline Loss of consciousness & $17(1.74)$ & $27(1.92)$ \\
\hline Tremor & $21(2.15)$ & $23(1.64)$ \\
\hline Transient ischaemic attack & $17(1.74)$ & $24(1.71)$ \\
\hline Muscular weakness & $18(1.85)$ & $22(1.56)$ \\
\hline Neuropathy peripheral & $16(1.64)$ & $22(1.56)$ \\
\hline Syncope & $14(1.44)$ & $18(1.28)$ \\
\hline Somnolence & $14(1.44)$ & $15(1.07)$ \\
\hline Balance disorder & $16(1.64)$ & $12(0.85)$ \\
\hline Seizure & $10(1.03)$ & $16(1.14)$ \\
\hline Mobility decreased & $10(1.03)$ & $13(0.92)$ \\
\hline Dementia & $16(1.64)$ & $6(0.43)$ \\
\hline Dysgeusia & $13(1.33)$ & $9(0.64)$ \\
\hline Migraine & $7(0.72)$ & $15(1.07)$ \\
\hline Vertigo & $9(0.92)$ & $13(0.92)$ \\
\hline Presyncope & $6(0.62)$ & $15(1.07)$ \\
\hline Other* & $247(25.31)$ & $377(23.75)$ \\
\hline Psychiatric disorders [no. of ADRs $=789$ ] & [no. of $A D R s=321]$ & [no. of $A D R s=468$ ] \\
\hline Insomnia & $38(11.84)$ & $41(8.76)$ \\
\hline Depression & $31(9.66)$ & $44(9.40)$ \\
\hline Confusional state & $28(8.72)$ & $39(8.33)$ \\
\hline Sleep disorder & $18(5.61)$ & $39(8.33)$ \\
\hline Disturbance in attention & $15(4.67)$ & $35(7.48)$ \\
\hline Anxiety & $20(6.23)$ & $29(6.20)$ \\
\hline Depressed mood & $21(6.54)$ & $20(4.27)$ \\
\hline Restlessness & $12(3.74)$ & $19(4.06)$ \\
\hline Apathy & $5(1.56)$ & $11(2.35)$ \\
\hline Nightmare & $7(2.18)$ & $9(1.92)$ \\
\hline Irritability & $7(2.18)$ & $8(1.71)$ \\
\hline Stress & $4(1.25)$ & $11(2.35)$ \\
\hline Suicidal ideation & $6(1.87)$ & $9(1.92)$ \\
\hline Disorientation & $4(1.25)$ & $9(1.92)$ \\
\hline Mental disorder & $5(1.56)$ & $8(1.71)$ \\
\hline Nervousness & $5(1.56)$ & $8(1.71)$ \\
\hline Hallucination & $4(1.25)$ & $8(1.71)$ \\
\hline Panic attack & $6(1.87)$ & $6(1.28)$ \\
\hline Dysarthria & $7(2.18)$ & $4(0.85)$ \\
\hline Sleep disorder due to a general medical condition & - & $11(2.35)$ \\
\hline Abnormal behaviour & $7(2.18)$ & $2(0.43)$ \\
\hline Abnormal dreams & $4(1.25)$ & $4(0.85)$ \\
\hline
\end{tabular}


Table 2 (continued)

\begin{tabular}{lll}
\hline System Organ Class & $\begin{array}{l}\text { Alirocumab } \\
\text { [no. of ICSRs = 856] }\end{array}$ & $\begin{array}{l}\text { Evolocumab } \\
\text { [no. of ICSRs = 1231] }\end{array}$ \\
\hline Aggression & $2(0.62)$ & $6(1.28)$ \\
Mood swings & $4(1.25)$ & $4(0.85)$ \\
Bradyphrenia & $2(0.62)$ & $5(1.07)$ \\
Other $^{\text {a }}$ & $59(18.30)$ & $79(16.79)$ \\
\hline
\end{tabular}

Data are expressed as $n(\%)$

ICSRs Individual Case Safety Reports, ADRs adverse drug reactions

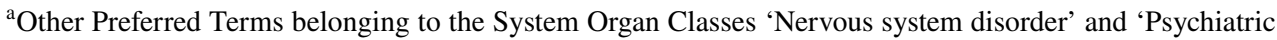
disorders' and reported in electronic supplementary Table 1
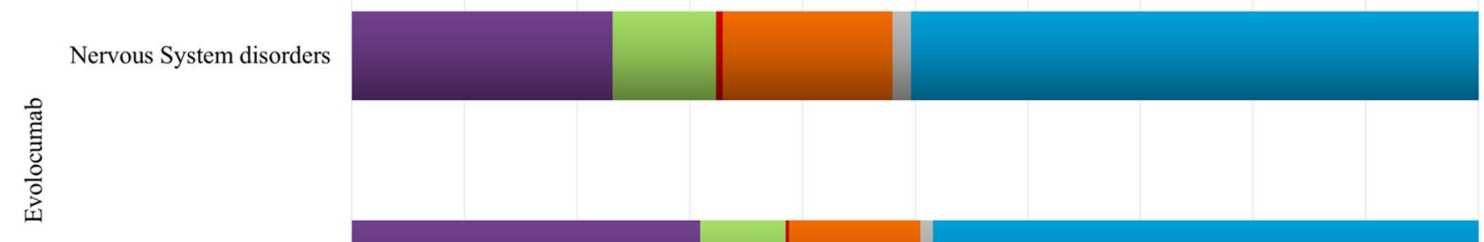

Psychiatric disorders
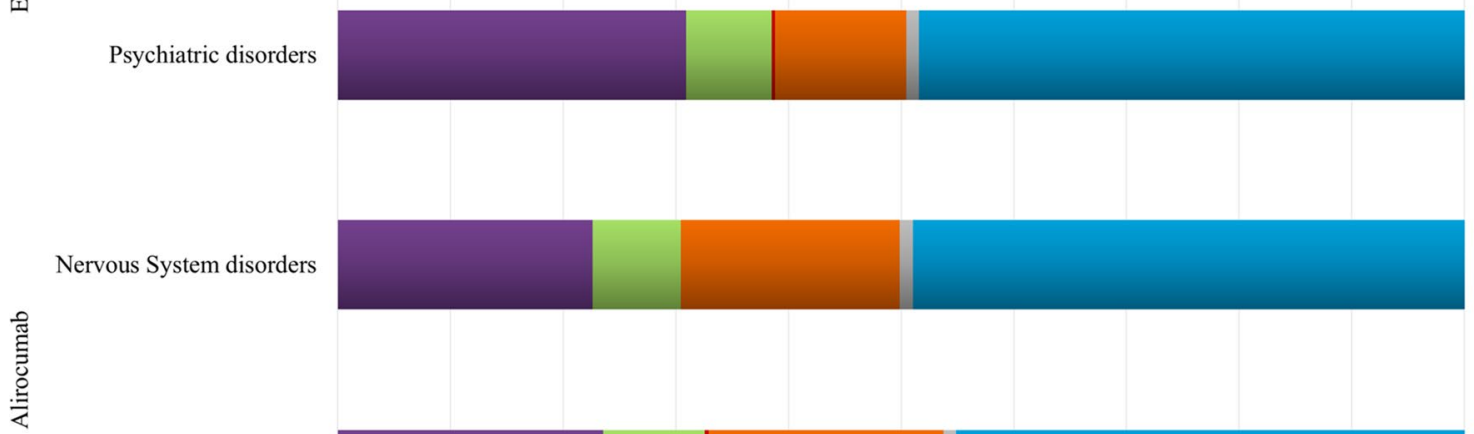

Psychiatric disorders

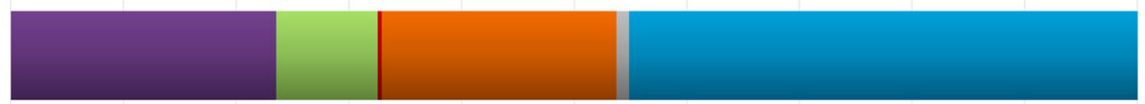

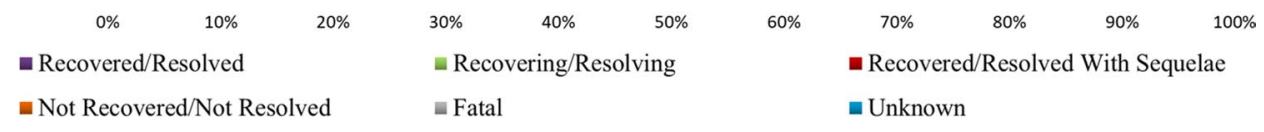

Fig. 2 Distribution of alirocumab and evolocumab-induced neuropsychiatric ADRs by outcome

We found that the median age of patients who experienced ADRs was 66 years, and 55\% were female. In line with our results, the literature suggests that the population treated with these drugs is typically older, often with comorbidities and concomitant medication use [22]. Furthermore, hypercholesterolaemia seems to be more common in women, particularly after menopause, which has been shown to be associated with an increase in total and LDL-cholesterol [32-39]. Lastly, it should be noted that, independently by drug classes, women are more susceptible to experience ADRs due to hormonal factors, as well as changes in pharmacokinetics [40-42].

Regarding distribution by seriousness, $66.6 \%$ of ICSRs reported ADRs that were considered as serious (this percentage was slightly higher for evolocumab). In contrast with this, as reported by a recent study based on a hospital registry and two pharmacovigilance databases, PCSK9Iinduced adverse events were usually mild and tended to resolve during follow-up [39]. In our opinion, the higher rate of serious ADRs that we found could be related to two main factors. First, neuropsychiatric ADRs can include different signs and symptoms, ranging from mild ADRs, including sleep disturbance, abnormal dreams, dizziness, loss of balance, and tinnitus, to more severe ADRs, such as depression, suicide, seizures and paralysis [43-45]. The second factor could be the source of ICSRs. Indeed, in our study, 72.3\% of ICSRs were reported by HCPs and, according to Inácio et al., these reporters seem to be especially prone to report 
Table 3 Distribution of Individual Case Safety Reports having alirocumab or evolocumab as the suspected drugs, by therapeutic indication

\begin{tabular}{llccc}
\hline Variable & Level & All ICSRs $[n=2041]$ & $\begin{array}{l}\text { ICSRs reporting alirocumab } \\
\text { as the suspect drug }[n=856]\end{array}$ & $\begin{array}{l}\text { ICSRs reporting evolocumab } \\
\text { as the suspect drug }[n=1231]\end{array}$ \\
\hline Therapeutic indication & $\begin{array}{l}\text { Lipid metabolism disorder } \\
\text { Cardiovascular disorders }\end{array}$ & $1423^{\mathrm{b}}(69.7)$ & $579^{\mathrm{b}}(67.6)$ & $870^{\mathrm{b}}(70.7)$ \\
& $176^{\mathrm{b}}(8.6)$ & $55^{\mathrm{b}}(6.4)$ & $123^{\mathrm{b}}(10)$ \\
& $\begin{array}{l}\text { Product used for unknown } \\
\text { indication }\end{array}$ & $635^{\mathrm{b}}(31.1)$ & $271^{\mathrm{b}}(31.6)$ & $386^{\mathrm{b}}(31.3)$ \\
& & $7^{\mathrm{b}}(0.8)$ & $11^{\mathrm{b}}(0.9)$ \\
\hline
\end{tabular}

Data are expressed as $n(\%)$

ICSRs Individual Case Safety Reports

${ }^{a}$ Other therapeutic indications: clinical trial participant; diabetes mellitus; disease risk factor; drug intolerance/drug hypersensitivity; hypolipidaemia; ill-defined disorder; intentional overdose; multiple sclerosis; plasmacytoma; product use in unapproved indication; routine health maintenance; dyspnea

${ }^{b}$ The sum of therapeutic indications reported in ICSRs is higher than the total number of ICSRs, since more than one therapeutic indication can be reported in each ICSR

Table 4 Reporting odds ratio of ICSRs with ADRs belonging to the SOCs 'Nervous system disorders' or 'Psychiatric disorders' for the comparison of alirocumab versus evolocumab

\begin{tabular}{lll}
\hline ICSRs & ROR $(95 \%$ CI $)$ & $p$ value \\
\hline Nervous system disorders & $1.02(0.91-1.14)$ & 0.720 \\
Psychiatric disorders & $1.12(0.94-1.34)$ & 0.187 \\
\hline
\end{tabular}

ICSRs Individual Case Safety Reports, ADRs adverse drug reactions, SOCs System Organ Classes, ROR reporting odds ratio, CI confidence interval

Table 5 Reporting odds ratio of ICSRs with nervous system disorders for the comparison of PCSK9Is versus statins

\begin{tabular}{lllr}
\hline PCSK9i & Statin & ROR $(95 \%$ CI $)$ & $p$ value \\
\hline Evolocumab & Atorvastatin & $1.55(1.44-1.68)$ & $<0.001$ \\
Evolocumab & Simvastatin & $1.12(1.02-1.22)$ & 0.013 \\
Evolocumab & Pravastatin & $1.11(0.98-1.26)$ & 0.098 \\
Evolocumab & Rosuvastatin & $1.00(0.92-1.09)$ & 0.976 \\
Evolocumab & Fluvastatin & $1.49(1.25-1.77)$ & $<0.001$ \\
Alirocumab & Atorvastatin & $1.58(1.45-1.74)$ & $<0.001$ \\
Alirocumab & Simvastatin & $1.14(1.03-1.26)$ & 0.011 \\
Alirocumab & Pravastatin & $1.13(0.99-1.29)$ & 0.067 \\
Alirocumab & Rosuvastatin & $1.02(0.92-1.13)$ & 0.676 \\
Alirocumab & Fluvastatin & $1.51(1.27-1.82)$ & $<0.001$ \\
\hline
\end{tabular}

ICSR Individual Case Safety Reports, PCSK9Is proprotein convertase subtilisin/kexin type 9 inhibitors, ROR reporting odds ratio, $C I$ confidence interval

more serious ADRs that result in hospitalization, are lifethreatening, or result in death [46]. The higher rate of ICSRs coming from HCPs is in line with findings from other studies [47-49]. Moreover, the new pharmacovigilance legislation has further increased the involvement of HCPs in pharmacovigilance activities [50-52].
Table 6 Reporting odds ratio of ICSRs with psychiatric disorders for the comparison of PCSK9Is versus statins

\begin{tabular}{lllr}
\hline PCSK9i & Statin & ROR $(95 \%$ CI $)$ & $p$ value \\
\hline Evolocumab & Atorvastatin & $0.96(0.85-1.09)$ & 0.546 \\
Evolocumab & Simvastatin & $0.56(0.49-0.64)$ & $<0.001$ \\
Evolocumab & Pravastatin & $0.61(0.51-0.73)$ & $<0.001$ \\
Evolocumab & Rosuvastatin & $0.68(0.60-0.78)$ & $<0.001$ \\
Evolocumab & Fluvastatin & $0.99(0.76-1.27)$ & 0.918 \\
Alirocumab & Atorvastatin & $1.08(0.94-1.25)$ & 0.272 \\
Alirocumab & Simvastatin & $0.63(0.54-0.73)$ & $<0.001$ \\
Alirocumab & Pravastatin & $0.69(0.57-0.83)$ & $<0.001$ \\
Alirocumab & Rosuvastatin & $0.76(0.66-0.89)$ & 0.001 \\
Alirocumab & Fluvastatin & $1.11(0.85-1.44)$ & 0.438 \\
\hline
\end{tabular}

ICSRs Individual Case Safety Reports, PCSK9Is proprotein convertase subtilisin/kexin type 9 inhibitors

In almost $40 \%$ of ICSRs, concomitant medications, especially acetylsalicylic acid, ezetimibe, levothyroxine, clopidogrel, atorvastatin and rosuvastatin, were reported. These data are not surprising considering that diseases for which those drugs are used are quite common in patients with hypercholesterolaemia, for whom multiple pharmacological treatments are frequently planned [53-60]. Therefore, the role of these concomitant medications in the occurrence of neuropsychiatric ADRs could not be excluded. Indeed, literature data suggest that psychiatric ADRs are associated with both statins and ezetimibe [61]. The observation that these lipid-lowering agents have similar adverse effects supports the hypothesis that decreased brain cell membrane cholesterol may be important in the etiology of psychiatric reactions. Furthermore, in a case report published in 2016, clopidogrel was associated with the occurrence of hallucinations [62]. Lastly, data on the role of aspirin in the occurrence of neuropsychiatric ADRs are quite conflicting. 
Indeed, some authors reported that salicylate intoxication could lead to psychosis and delirium [63, 64]. Other studies have suggested that aspirin might have beneficial effects in patients with dementia, by reducing the risk of recurrent vascular events. In addition, high-dose aspirin $(325 \mathrm{mg} /$ day) was associated with reduced rates of Alzheimer's disease $[65,66]$. Lastly, the concomitant use of multiple drugs could increase the occurrence of ADRs, also as a result of drug-drug interactions (DDIs), even though, to our knowledge, no data on DDIs between PCSK9Is and other drugs currently exist.

The RORs of ICSRs with ADRs belonging to the SOCs 'Nervous system disorders' or 'Psychiatric disorders' showed no difference between alirocumab and evolocumab. Currently, studies that have directly compared alirocumab and evolocumab, in terms of efficacy and safety profile, are mainly represented by network meta-analysis. According to the results of a recent systematic review and network metaanalysis of 30 clinical trials (59,026 patients), no significant differences between alirocumab and evolocumab in myocardial infarction, stroke, coronary revascularization, treatment discontinuations, systemic allergic reaction, neurocognitive events, ophthalmologic events, or new-onset of or worsening of pre-existing diabetes were found. Alirocumab was associated with an increased risk of injection site reaction compared with evolocumab [67]. In addition, the results of a further meta-analysis of 39 clinical trials (66,478 patients, of whom 14,639 were treated with alirocumab, 21,257 were treated with evolocumab, and 30,582 were controls) showed that the use of both PCSK9Is was not associated with increased risk of neurocognitive adverse events $(p=0.91)$ [68]. Based on the same results, Bajaj et al. concluded that PCSK9Is have neuroprotective effects due to the decrease in ischaemic-mediated neurovascular events [69]. Harvey et al. recently carried out a meta-analysis of individual patient data $(3340$ patients treated with alirocumab $75 / 150 \mathrm{mg}$ every 2 weeks, 1276 treated with placebo, and 618 treated with ezetimibe). Neurocognitive ADRs were observed in $0.9 \%$ of alirocumab-treated patients versus $0.7 \%$ of placebotreated patients, and in $1.2 \%$ of alirocumab-treated patients versus $1.3 \%$ of ezetimibe-treated patients. The incidence of neurocognitive ADRs was similar between alirocumab and controls when stratified by age. Up to 104 weeks, no significant difference was found in the incidence of neurocognitive ADRs between the alirocumab and control groups [70]. Giugliano et al. have recently evaluated the effect of evolocumab on cognitive function in 1204 patients (mean age 65 years) who were followed for 1.6 years without evidencing any association with adverse cognitive effects [71]. Despite the fact that the lack of major safety concerns with PCSK9Is found in both the FOURIER and ODYSSEY OUTCOMES trials is encouraging, several important questions remain to be answered. In particular, more data on the long-term effects of PCSK9Is are strongly needed [72, 73 ] and postmarket surveillance data could provide additional relevant long-term safety data. Open-label extension studies from previously published trials on PCSK9Is are underway and can also address potentially long-term benefits, at the same time ruling out possible long-term harms (NCT02867813, NCT03080935, NCT01439880, NCT01954394, NCT01854918). Furthermore, a dedicated study for neurocognitive evaluation is ongoing in a separate study in the ODYSSEY OUTCOMES trial.

Therefore, according to the above results, no evidence on the effects on cognitive function was found for alirocumab or evolocumab. As recently reported by Adorni et al., neither preclinical nor clinical studies have demonstrated a correlation between low levels of LDL-cholesterol and neurocognitive effects. Furthermore, the BBB limits the access of both PCSK9Is to the central nervous system. Therefore, if the BBB is intact, the penetration of alirocumab and evolocumab is estimated to be about $0.1 \%$ [74]. Lastly, Paciullo et al. recently speculated about the possible pleiotropic effects of PCSK9Is on platelet activity and coagulation factors. Specifically, the authors reported that in patients with acute coronary syndrome treated with P2Y12 inhibitors, a direct association between serum PCSK9 levels and residual platelet reactivity was found. Furthermore, blood coagulation factor VIII (FVIII) is eliminated from circulation by members of the LDLR family; given that PCSK9 degrades LDLRs, it is conceivable that PCSK9Is, by enhancing LDLR expression, may reduce circulating FVIII, thus contributing to the prevention of cardiovascular events [75].

Lastly, while evolocumab and alirocumab showed a higher reporting probability of ICSRs with neurologic ADRs compared with atorvastatin and fluvastatin, they showed a lower reporting probability of ICSRs with psychiatric ADRs compared with simvastatin, pravastatin, and rosuvastatin. Although our data are apparently in contrast with the literature data in terms of the potency of statins, statins may induce both psychiatric and neurological ADRs [76, 77], which seem to increase when the level of cholesterol and omega-3 fatty acid are reduced (both conditions are associated with statins). Data from a meta-analysis of 11 clinical studies found that neurocognitive events occurred in $0.8 \%$ of patients receiving PCSK9Is versus $0.5 \%$ of patients not receiving PCSK9Is [78]. Since, to our knowledge, no other studies have previously compared the risk of neuropsychiatric ADRs between statins and PCSK9Is, no firm conclusion can be drawn regarding our findings, which need to be confirmed by ad hoc pharmacoepidemiology studies.

\subsection{Strengths and Limitations}

To our knowledge, this is the first study that has analyzed the safety profile of PCSK9Is in terms of neurocognitive 
adverse events, using data from the spontaneous reporting system on a European level. Considering the recent authorization of these drugs, the specificity of our analysis, and given the lack of safety data from the real-life context, our study represents the first comprehensive evaluation of safety data related to PCSK9Is, using data from the Eudravigilance database. Furthermore, we have analyzed a huge amount of data providing statistical comparison, ROR, 95\% CI, and Chi-square test, between alirocumab and evolocumab, and a lower/higher probability of reporting neuropsychiatric ADRs. Furthermore, data from the spontaneous reporting system contribute to a better characterization of the drug safety profiles, especially for newly authorized medicines and in the frail population, including the elderly and patients with multiple comorbidities, which are usually excluded by premarketing clinical trials. Therefore, data obtained from the spontaneous reporting system represent one of the main sources of information for these populations.

Despite its strengths, the spontaneous reporting system is affected by limitations that are mainly related to the underreporting and inaccuracy or incompleteness of information. Therefore, we cannot exclude that important information was not listed in ICSRs that we have evaluated (i.e. degrees of seriousness and outcome, previous/current medical conditions, further suspected or concomitant medications). All information that was not reported could have potentially affected the occurrence of ADRs that we have analyzed. Another important limitation related to our data source was the impossibility to compute the ROR only for ICSRs related to the impairment of cognitive function, as not all ICSRs classified into nervous system disorders or psychiatric disorders may be relevant to impaired cognitive function. Finally, even though in our study ICSRs were individually reviewed, considering also data on other drugs that could have potentially induced neuropsychiatric ADRs, the risk of notoriety bias cannot be excluded.

\section{Conclusion}

Using data from the Eudravigilance database, we carried out a descriptive analysis of ICSRs related to alirocumab and evolocumab and reporting neurological and psychiatric ADRs. Our results have demonstrated that $22.7 \%$ of all ICSRs reporting alirocumab or evolocumab as suspect described the occurrence of neuropsychiatric ADRs. The majority of reported ADRs were serious and were associated with female sex. Moreover, alirocumab was associated with an increased reporting probability of nervous and psychiatric ADRs compared with evolocumab.

Considering that both drugs have recently obtained marketing authorization, further studies are strongly needed to evaluate the safety profile of PCSK9Is on neurocognitive function, and, specifically, data from real-life contexts needs to be collected. Indeed, such adverse events may have a strong impact on the quality of life of patients, especially the elderly, and also the economic burden of such treatments. In this context, we believe that an effective pharmacovigilance system would be able to guarantee continuous monitoring of the safety profile of these drugs, playing a key role in the real definition of the risk/benefit profile of the PCSK9Is, ensuring proper use of these medications, and providing ideas and directions for future research in order to confirm or confute any possible association between a drug and an adverse event, such as those that we have evaluated.

\section{Declarations}

Funding Open access funding provided by Universitá degli Studi della Campania Luigi Vanvitelli within the CRUI-CARE Agreement.

Conflict of interest The authors declare that they have no conflict of interest.

Ethics approval Safety data deriving from the spontaneous reporting system are anonymous and in compliance with the ethical standard. Therefore, no further ethical measure was required.

Consent to participate Not Applicable.

Consent for publication All authors agreed with the publication of study's results.

Availability of data and material The dataset generated for this study was obtained from the Eudravigilance database (available at the following link: http://www.adrreports.eu/en/search.html)

Code availability Not applicable.

Author contributions GdM, AZ, CS, AM, MG, LS, CF, CR, FR and AC made substantial contributions to the acquisition, analysis, or interpretation of data for the work; drafted the work and revised it for important intellectual content; wrote the paper; agreed to be accountable for all aspects of the work in ensuring that questions related to the accuracy or integrity of any part of the work are appropriately investigated and resolved; and approved the final version of the manuscript to be published. GdM, AZ, CS, FR and AC developed the concept.

Open Access This article is licensed under a Creative Commons Attribution-NonCommercial 4.0 International License, which permits any non-commercial use, sharing, adaptation, distribution and reproduction in any medium or format, as long as you give appropriate credit to the original author(s) and the source, provide a link to the Creative Commons licence, and indicate if changes were made. The images or other third party material in this article are included in the article's Creative Commons licence, unless indicated otherwise in a credit line to the material. If material is not included in the article's Creative Commons licence and your intended use is not permitted by statutory regulation or exceeds the permitted use, you will need to obtain permission directly from the copyright holder. To view a copy of this licence, visit http://creativecommons.org/licenses/by-nc/4.0/. 


\section{References}

1. Virmani R, Kolodgie FD, Burke AP, Farb A, Schwartz SM. Lessons from sudden coronary death: a comprehensive morphological classification scheme for atherosclerotic lesions. Arterioscler Thromb Vasc Biol. 2000;20(5):12621-75. https://doi. org/10.1161/01.atv.20.5.1262.

2. Ogura M. PCSK9 inhibition in the management of familial hypercholesterolemia. J Cardiol. 2018;71(1):1-7. https://doi. org/10.1016/j.jjcc.2017.07.002.

3. Abifadel M, Varret M, Rabès JP, Allard D, Ouguerram K, Devillers M, et al. Mutations in PCSK9 cause autosomal dominant hypercholesterolemia. Nat Genet. 2003;34(2):154-6. https://doi. org/10.1038/ng1161.

4. Horton JD, Cohen JC, Hobbs HH. PCSK9: a convertase that coordinates LDL catabolism. J Lipid Res. 2009;50(Suppl):S172-7. https://doi.org/10.1194/jlr.R800091-JLR200.

5. Seidah NG, Benjannet S, Wickham L, Marcinkiewicz J, Jasmin $\mathrm{SB}$, Stifani S, et al. The secretory proproteinconvertase neural apoptosis-regulated convertase 1 (NARC-1): liver regeneration and neuronal differentiation. Proc Natl Acad Sci USA. 2003;100(3):928-33. https://doi.org/10.1073/pnas.0335507100.

6. Bonaca MP, Nault P, Giugliano RP, Keech AC, Pineda AL, Kanevsky E, et al. Low-density lipoprotein cholesterol lowering with evolocumab and outcomes in patients with peripheral artery disease: insights from the FOURIER trial (further cardiovascular outcomes research with PCSK9 inhibition in subjects with elevated risk). Circulation. 2018;137(4):338-50. https://doi. org/10.1161/CIRCULATIONAHA.117.032235.

7. Schwartz GG, Bessac L, Berdan LG, Bhatt DL, Bittner V, Diaz $\mathrm{R}$, et al. Effect of alirocumab, a monoclonal antibody to PCSK9, on long-term cardiovascular outcomes following acute coronary syndromes: rationale and design of the ODYSSEY outcomes trial. Am Heart J. 2014;168(5):682-9. https://doi.org/10.1016/j. ahj.2014.07.028.

8. Page MM, Watts GF. Emerging PCSK9 inhibitors for treating dyslipidaemia: buttressing the gaps in coronary prevention. Expert Opin Emerg Drugs. 2015;20(2):299-312. https://doi. org/10.1517/14728214.2015.1035709.

9. Bai J, Gong LL, Li QF, Wang ZH. Long-term efficacy and safety of proprotein convertase subtilisin/kexin 9 monoclonal antibodies: a meta-analysis of 11 randomized controlled trials. J Clin Lipidol. 2018;12(2):277-291.e3. https://doi.org/10.1016/j. jacl.2018.01.004.

10. Chen Q, Wu G, Li C, Qin X, Liu R, Zhang M. Safety of proprotein convertase subtilisin/kexin type 9 monoclonal antibodies in regard to diabetes mellitus: a systematic review and metaanalysis of randomized controlled trials. Am J Cardiovasc Drugs. 2020;20(4):343-53. https://doi.org/10.1007/s40256-019-00386-w.

11. Muldoon MF, Barger SD, Ryan CM, Flory JD, Lehoczky JP, Matthews KA, et al. Effects of lovastatin on cognitive function and psychological well-being. Am J Med. 2000;108(7):538-46. https ://doi.org/10.1016/s0002-9343(00)00353-3.

12. Muldoon MF, Ryan CM, Sereika SM, Flory JD, Manuck SB. Randomized trial of the effects of simvastatin on cognitive functioning in hypercholesterolemic adults. Am J Med. 2004;117(11):8239. https://doi.org/10.1016/j.amjmed.2004.07.041.

13. Swiger KJ, Martin SS. PCSK9 inhibitors and neurocognitive adverse events: exploring the FDA directive and a proposal for N-of-1 trials. Drug Saf. 2015;38(6):519-26. https://doi. org/10.1007/s40264-015-0296-6.

14. Picard C, Poirier A, Bélanger S, Labonté A, Auld D, Poirier J, the PREVENT-AD Research Group. Proprotein convertase subtilisin/kexin type 9 (PCSK9) in Alzheimer's disease: a genetic and proteomic multi-cohort study. PLoS ONE. 2019;14(8):e0220254. https://doi.org/10.1371/journal.pone.02202 54.

15. de Bruijn RF, Ikram MA. Cardiovascular risk factors and future risk of Alzheimer's disease. BMC Med. 2014;12:130. https://doi. org/10.1186/s12916-014-0130-5.

16. Leritz EC, McGlinchey RE, Kellison I, Rudolph JL, Milberg WP. Cardiovascular disease risk factors and cognition in the elderly. Curr Cardiovasc Risk Rep. 2011;5(5):407-12. https:// doi.org/10.1007/s12170-011-0189-x.

17. The Wall Street Journal. FDA advises of adverse effects from new cholesterol drugs. http://www.wsj.com/articles/SB10001424 052702304732804579425612487904436. Accessed 20 Feb 2015.

18. Schwartz GG, Steg PG, Szarek M, Bhatt DL, Bittner VA, Diaz R, et al. Alirocumab and cardiovascular outcomes after acute coronary syndrome. N Engl J Med. 2018;379(22):2097-107. https:// doi.org/10.1056/NEJMoa1801174.

19. Sabatine MS, Giugliano RP, Wiviott SD, Raal FJ, Blom DJ, Robinson J, et al. Efficacy and safety of evolocumab in reducing lipids and cardiovascular events. N Engl J Med. 2015;372(16):1500-9. https://doi.org/10.1056/NEJMoa1500858.

20. Sabatine MS, Giugliano RP, Keech AC, Honarpour N, Wiviott SD, Murphy SA, et al. Evolocumab and Clinical Outcomes in Patients with Cardiovascular Disease. N Engl J Med. 2017;376(18):171322. https://doi.org/10.1056/NEJMoa1615664.

21. Robinson JG, Farnier M, Krempf M, Bergeron J, Luc G, Averna $\mathrm{M}$, et al. Efficacy and safety of alirocumab in reducing lipids and cardiovascular events. N Engl J Med. 2015;372(16):148999. https://doi.org/10.1056/NEJMoa1501031.

22. Giugliano RP, Mach F, Zavitz K, Kurtz C, Im K, Kanevsky E, et al. Cognitive function in a randomized trial of evolocumab. $\mathrm{N}$ Engl J Med. 2017;377(7):633-43. https://doi.org/10.1056/NEJMo a1701131.

23. Björkhem I, Meaney S. Brain cholesterol: long secret life behind a barrier. Arterioscler Thromb Vasc Biol. 2004;24(5):806-15. https ://doi.org/10.1161/01.ATV.0000120374.59826.1b.

24. Joseph JA, Denisova N, Villalobos-Molina R, Erat S, Strain J. Oxidative stress and age-related neuronal deficits. Mol Chem Neuropathol. 1996;28(1-3):35-40. https://doi.org/10.1007/BF028 15202.

25. Wahler JB, Swain MG, Carson R, Bergasa NV, Jones EA. Bloodbrain barrier permeability is markedly decreased in cholestasis in the rat. Hepatology. 1993;17(6):1103-8.

26. Jonas MC, Costantini C, Puglielli L. PCSK9 is required for the disposal of non-acetylated intermediates of the nascent membrane protein BACE1. EMBO Rep. 2008;9(9):916-22. https:// doi.org/10.1038/embor.2008.132.

27. Liu M, Wu G, Baysarowich J, Kavana M, Addona GH, Bierilo $\mathrm{KK}$, et al. PCSK9 is not involved in the degradation of LDL receptors and BACE1 in the adult mouse brain. J Lipid Res. 2010;51(9):2611-8. https://doi.org/10.1194/jlr.M006635.

28. Kivipelto M, Helkala EL, Laakso MP, Hänninen T, Hallikainen M, Alhainen K, et al. Midlife vascular risk factors and Alzheimer's disease in later life: longitudinal, population based study. BMJ. 2001;322(7300):1447-51. https://doi.org/10.1136/ bmj.322.7300.1447.

29. Koren MJ, Giugliano RP, Raal FJ, Sullivan D, Bolognese M, Langslet G, et al. Efficacy and safety of longer-term administration of evolocumab (AMG 145) in patients with hypercholesterolemia: 52-week results from the Open-Label Study of Long-Term Evaluation Against LDL-C (OSLER) randomized trial. Circulation. 2014;129(2):234-43. https://doi.org/10.1161/CIRCULATIO NAHA.113.007012.

30. Piccinni C, Antonazzo IC, Maggioni AP, Pedrini A, Calabria S, Ronconi G, et al. PCSK9 inhibitors' new users: analysis of prescription patterns and patients' characteristics from an Italian 
real-world study. Clin Drug Investig. 2020;40(2):173-81. https:// doi.org/10.1007/s40261-019-00877-3.

31. Chamberlain AM, Gong Y, Shaw KM, Bian J, Song WL, Linton MF, et al. PCSK9 inhibitor use in the real world: data from the national patient-centered research network. J Am Heart Assoc. 2019;8(9):e011246. https://doi.org/10.1161/JAHA.118.011246.

32. Karalis DG, Mallya UG, Ghannam AF, Elassal J, Gupta R, Boklage SH. Prescribing patterns of proproteinconvertasesubtilisin-kexin type 9 inhibitors in eligible patients with clinical atherosclerotic cardiovascular disease or heterozygous familial hypercholesterolemia. Am J Cardiol. 2018;121(10):1155-61. https ://doi.org/10.1016/j.amjcard.2018.02.002.

33. Tai MH, Shepherd J, Bailey H, Williams N, Hatz M, Campos Tapias I, Catterick D, Worth G. Real-world treatment patterns of PCSK9 inhibitors among patients with dyslipidemia in Germany, Spain, and the United Kingdom. Curr Med Res Opin. 2019;35(5):829-35. https://doi.org/10.1080/03007 995.2018 .1532885$.

34. Cífková R, Krajčoviechová A. Dyslipidemia and cardiovasculardisease in women. Curr Cardiol Rep. 2015;17(7):609. https://doi. org/10.1007/s11886-015-0609-5.

35. Phan BA, Toth PP. Dyslipidemia in women: etiology and management. Int J Womens Health. 2014;6:185-94. https://doi. org/10.2147/IJWH.S38133.

36. Kolovou GD, Anagnostopoulou KK, Damaskos DS, Bilianou HI, Mihas C, Milionis HJ, et al. Gender differences in the lipid profile of dyslipidemic subjects. Eur J Intern Med. 2009;20(2):145-51. https://doi.org/10.1016/j.ejim.2008.06.011.

37. Wang X, Magkos F, Mittendorfer B. Sex differences in lipid and lipoprotein metabolism: it's not just about sex hormones. J Clin Endocrinol Metab. 2011;96(4):885-93. https://doi.org/10.1210/ jc.2010-2061.

38. Baars A, Oosting A, Lohuis M, Koehorst M, El Aidy S, Hugenholtz F, et al. Sex differences in lipid metabolism are affected by presence of the gut microbiota. Sci Rep. 2018;8(1):13426. https ://doi.org/10.1038/s41598-018-31695-w.

39. Gürgöze MT, Muller-Hansma AHG, Schreuder MM, GalemaBoers AMH, Boersma E, Roeters van Lennep JE. Adverse events associated with PCSK9 inhibitors: a real-world experience. Clin Pharmacol Ther. 2019;105(2):496-504. https://doi.org/10.1002/ cpt.1193.

40. Mascolo A, Ruggiero R, Sessa M, Scavone C, Sportiello L, Rafaniello $\mathrm{C}$, et al. Preventable cases of oral anticoagulant-induced bleeding: data from the spontaneous reporting system. Front Pharmacol. 2019;10:425. https://doi.org/10.3389/fphar.2019.00425.

41. Scavone C, Di Mauro C, Ruggiero R, Bernardi FF, Trama U, Aiezza ML, et al. Severe cutaneous adverse drug reactions associated with allopurinol: an analysis of spontaneous reporting system in Southern Italy. Drugs Real World Outcomes. 2020;7(1):41-51. https://doi.org/10.1007/s40801-019-00174-7.

42. Scavone C, Rafaniello C, Brusco S, Bertini M, Menditto E, Orlando V, et al. Did the new Italian law on mandatory vaccines affect adverse event following immunization's reporting? A pharmacovigilance study in Southern Italy. Front Pharmacol. 2018;9:1003. https://doi.org/10.3389/fphar.2018.01003.

43. Nevin RL, Leoutsakos JM. Identification of a syndrome class of neuropsychiatric adverse reactions to mefloquine from latent class modeling of FDA adverse event reporting system data. Drugs R D. 2017;17(1):199-210. https://doi.org/10.1007/s40268-016-0167-3.

44. Drug-Induced Diseases. Section IV: Drug-Induced Psychiatric Diseases Chapter 18: depression Sheila Botts and Melody Ryan. https://www.ashp.org/-/media/assets/pharmacy-practice/ resource-centers/medications-suicidality/drug-induced-diseases. ash $\mathrm{P}$ la $=$ en \&hash $=3 \mathrm{~F} 47 \mathrm{~B} 710 \mathrm{CD} 1 \mathrm{AEF} 5 \mathrm{~F} 8 \mathrm{~A} 970 \mathrm{BEF} 014 \mathrm{EF} 1$ 6FC8115FC7. Accessed 12 Oct 2020.
45. Demler TL. Drug-Induced Neurologic Conditions. US Pharm. 2014;39(1):47-51. https://www.uspharmacist.com/article/drugi nduced-neurologic-conditions. Accessed 12 Oct 2020.

46. Inácio $\mathrm{P}$, Cavaco $\mathrm{A}$, Airaksinen $\mathrm{M}$. The value of patient reporting to the pharmacovigilance system: a systematic review. $\mathrm{Br} \mathrm{J}$ Clin Pharmacol. 2017;83(2):227-46. https://doi.org/10.1111/ bcp. 13098 .

47. Güner MD, Ekmekci PE. Healthcare professionals' pharmacovigilance knowledge and adverse drug reaction reporting behavior and factors determining the reporting rates. J Drug Assess. 2019;8(1):13-20. https://doi.org/10.1080/21556660.2019.15661 37.

48. Kassa Alemu B, Biru TT. Health Care Professionals' knowledge, attitude, and practice towards adverse drug reaction reporting and associated factors at selected public Hospitals in Northeast Ethiopia: a cross-sectional study. Biomed Res Int. 2019;2019:8690546. https://doi.org/10.1155/2019/8690546.

49. Kc S, Tragulpiankit P, Gorsanan S, Edwards IR. Attitudes among healthcare professionals to the reporting of adverse drug reactions in Nepal. BMC Pharmacol Toxicol. 2013;14:16. https://doi. org/10.1186/2050-6511-14-16.

50. Santoro A, Genov G, Spooner A, Raine J, Arlett P. Promoting and protecting public health: how the European union pharmacovigilance system works. Drug Saf. 2017;40(10):855-69. https://doi. org/10.1007/s40264-017-0572-8.

51. European Medicines Agency. Guideline on good pharmacovigilance practices (GVP). 2012. https://www.ema.europa.eu/en/ documents/scientific-guideline/draft-guideline-good-pharmacovi gilance-practices-module-vi-management-reporting-adverse-react ions_en.pdf. Accessed 12 Oct 2020.

52. Russo E, Mazzitello C, Esposito S, De Francesco A, Capuano A, De Sarro G. Pharmacovigilance in Italy: an overview. J Pharmacol Pharmacother. 2013;4(Suppl 1):S20-S28. https://doi. org/10.4103/0976-500x.120942.

53. Luzak B, Boncler M, Rywaniak J, Wilk R, Stanczyk L, Czyz M, et al. The effect of a platelet cholesterol modulation on the acetylsalicylic acid-mediated blood platelet inhibition in hypercholesterolemic patients. Eur J Pharmacol. 2011;658(2-3):91-7. https ://doi.org/10.1016/j.ejphar.2011.02.026.

54. Mauro VF, Tuckerman CE. Ezetimibe for management of hypercholesterolemia. Ann Pharmacother. 2003;37(6):839-48. https:// doi.org/10.1345/aph.1c209.

55. Lee YK, Lee H, Han S, Jung H, Shin DY, Nam K-H, et al. Association between thyroid-stimulating hormone level after total thyroidectomy and hypercholesterolemia in female patients with differentiated thyroid cancer: a retrospective study. J Clin Med. 2019;8(8):1106. https://doi.org/10.3390/jcm8081106.

56. De Kam P-J, Luo W-L, Wenning L, Ratcliffe L, Sisk CM, Royalty $\mathrm{J}$, et al. The effects of laropiprant on the antiplatelet activity of coadministered clopidogrel and aspirin. Platelets. 2014;25(7):480-7. https://doi.org/10.3109/09537104.2013.836747.

57. Hirsh J, Bhatt DL. Comparative benefits of clopidogrel and aspirin in high-risk patient populations. Arch Intern Med. 2004;164(19):2106-10. https://doi.org/10.1001/archi nte.164.19.2106.

58. Ya-Bin M, Chan P, Zhang Y, Tomlinson B. Evaluating the efficacy and safety of atorvastatin + ezetimibe in a fixed-dose combination for the treatment of hypercholesterolemia. Expert Opin Pharmacother. 2019;20(8):917-28. https://doi.org/10.1080/14656 566.2019 .1594776$.

59. Zhao S, Peng D. Efficacy and safety of rosuvastatin versus atorvastatin in high-risk Chinese patients with hypercholesterolemia: a randomized, double-blind, active-controlled study. Curr Med Res Opin. 2018;34(2):227-35. https://doi.org/10.1080/03007 995.2017.1371584. 
60. Kim W, Yoon YE, Shin S-H, Bae J-W, Hong B-K, Hong SJ, et al. Efficacy and safety of ezetimibe and rosuvastatin combination therapy versus those of rosuvastatin monotherapy in patients with primary hypercholesterolemia. Clin Ther. 2018;40(6):993-1013. https://doi.org/10.1016/j.clinthera.2018.04.015.

61. Tatley M, Savage R. Psychiatric adverse reactions with statins, fibrates and ezetimibe: implications for the use of lipid-lowering agents. Drug Saf. 2007;30(3):195-201. https://doi. org/10.2165/00002018-200730030-00003.

62. Osuagwu FC, Parashar S, Amalraj B, Tinklepaugh M, Dillon J, Bradley RH. Clopidogrel-induced auditory and visual hallucinations. Prim Care Companion CNS Disord. 2016;18(3). https://doi. org/10.4088/PCC.15101894.

63. Good AE, Welch MH. Hospital-acquired salicylate intoxication report of a case with psychosis, acidosis, and coma. J Rheumatol. 1975;2(1):52-60.

64. Sawrer-Foner GJ, Morrison GH. Acute toxic psychosis due to acetyl-salicylicacid. Can Serv Med J. 1955;11(9):599-606.

65. Williams PS, Rands G, Orrel M, Spector A. Aspirin for vascular dementia. Cochrane Database Syst Rev. 2000;(4):CD001296. https://doi.org/10.1002/14651858.CD001 296.

66. Nilsson SE, Johansson B, Takkinen S, Berg S, Zarit S, McClearn $\mathrm{G}$, et al. Does aspirin protect against Alzheimer's dementia? A study in a Swedish population-based sample aged $>$ or $=80$ years. Eur J Clin Pharmacol. 2003;59(4):313-9. https://doi.org/10.1007/ s00228-003-0618-y.

67. Guedeney P, Sorrentino S, Giustino G, Chapelle C, Laporte S, Claessen BE, et al. Indirect comparison of the efficacy and safety of alirocumab and evolocumab: a systematic review and network meta-analysis. Eur Heart J Cardiovasc Pharmacother. 2020. pvaa024. https://doi.org/10.1093/ehjcvp/pvaa024.

68. Guedeney P, Giustino G, Sorrentino S, Claessen BE, Camaj A, Kalkman DN, et al. Efficacy and safety of alirocumab and evolocumab: a systematic review and meta-analysis of randomized controlled trials. Eur Heart J. 2019; ehz430. https://doi. org/10.1093/eurheartj/ehz430.

69. Bajaj NS, Patel N, Kalra R, Ahmad A, Venkatraman A, Arora G, et al. Neurological effects of proproteinconvertasesubtilisin/kexin type 9 inhibitors: direct comparisons. Eur Heart J Qual Care Clin Outcomes. 2018;4(2):132-41. https://doi.org/10.1093/ehjqcco/ qcx037.
70. Harvey PD, Sabbagh MN, Harrison JE, Ginsberg HN, Chapman MJ, Manvelian G, et al. No evidence of neurocognitive adverse events associated with alirocumab treatment in 3340 patients from 14 randomized Phase 2 and 3 controlled trials: a meta-analysis of individual patient data. Eur Heart J. 2018;39(5):374-81. https:// doi.org/10.1093/eurheartj/ehx661.

71. Giugliano RP, Sabatine MS, Ott BR. Cognitive function in a randomized trial of evolocumab. N Engl J Med. 2017;377(7):633-43. https://doi.org/10.1056/NEJMoa1701131.

72. Cohen JC, Boerwinkle E, Mosley TH Jr, Hobbs HH. Sequence variations in PCSK9, low LDL, and protection against coronary heart disease. N Engl J Med. 2006;354(12):1264-72. https://doi. org/10.1056/NEJMoa054013.

73. Koren MJ, Sabatine MS, Giugliano RP, Langslet G, Wiviott SD, Ruzza A, et al. Long-Term Efficacy and Safety of Evolocumab in Patients With Hypercholesterolemia. J Am Coll Cardiol. 2019;74(17):2132-46. https://doi.org/10.1016/j. jacc.2019.08.1024.

74. Adorni MP, Ruscica M, Ferri N, Bernini F, Zimetti F. Proprotein convertase subtilisin/kexin type 9 , brain cholesterol homeostasis and potential implication for Alzheimer's disease. Front Aging Neurosci. 2019;11:120. https://doi.org/10.3389/fnagi.2019.00120.

75. Paciullo F, Momi S, Gresele P. PCSK9 in haemostasis and thrombosis: possible pleiotropic effects of PCSK9 inhibitors in cardiovascular prevention. Thromb Haemost. 2019;359-67. https://doi. org/10.1055/s-0038-1676863.

76. Tuccori M, Montagnani S, Mantarro S, Capogrosso-Sansone A, Ruggiero E, Saporiti A, et al. Neuropsychiatric adverse events associated with statins: epidemiology, pathophysiology, prevention and management. CNS Drugs. 2014;28(3):249-72. https:// doi.org/10.1007/s40263-013-0135-1 (PMID: 24435290).

77. Cham S, Koslik HJ, Golomb BA. Mood, personality, and behavior changes during treatment with statins: a case series. Drug Saf Case Rep. 2016;3(1):1. https://doi.org/10.1007/s40800-015-0024-2.

78. Khan AR, Bavishi C, Riaz H, Farid TA, Khan S, Atlas M, et al. Increased risk of adverse neurocognitive outcomes with proprotein convertase subtilisin-kexin type 9 inhibitors. Circ Cardiovasc Qual Outcomes. 2017;10(1):e003153. https://doi.org/10.1161/ CIRCOUTCOMES.116.003153. 